\title{
Apparent foreign-body-like structure detected in the left atrium after catheter ablation for persistent atrial fibrillation
}

\author{
Tomas Skala ${ }^{\mathrm{a}}$, Ondrej Moravec ${ }^{\mathrm{a}}$, Martin Hutyraa ${ }^{\mathrm{a}}$, Zbynek Tudos ${ }^{\mathrm{b}}$, Martin Kocher ${ }^{\mathrm{b}}$, Milos Taborsky ${ }^{\mathrm{a}}$
}

Background. A foreign body left in left atrium after catheter ablation of atrial fibrillation is a very rare complication. Nevertheless, there are no reports so far about a large newly emerged structure only giving the impression of being a foreign body left in the heart after catheter ablation.

Case report. This report presents a case of a patient after catheter ablation for persistent atrial fibrillation. Because of atrial tachycardia during follow-up, patient was indicated for reablation. Imaging methods including intracardiac echocardiography showed a straw-like foreign body with the character of a transseptal sheath in left atrium. A cardiac surgery was performed with extraction of the foreign body. We found a fibrous chord-like material in the left atrium, microscopy showed myocardial tissue with continuous transition to fibrinous elastic vessel. No signs of foreign material were found.

Conclusion. We have found a newly emerged body giving the impression of being of foreign origin in left atrium after catheter ablation of atrial fibrillation. Microscopy of the extracted material showed myocardial tissue with continuous transition to fibrinous elastic vessel and a fibrinous tissue with focal dystrophic calcification. Its origin remains unknown.

Key words: catheter ablation, atrial fibrillation, cardiac surgery, foreign material, fibrinous tissue

Received: March 16, 2018; Accepted: August 31, 2018; Available online: September 11, 2018

https://doi.org/10.5507/bp.2018.052

${ }^{a}$ Department of Internal Medicine I - Cardiology, University Hospital Olomouc, Czech Republic

${ }^{b}$ Department of Radiology, University Hospital Olomouc, Czech Republic

Corresponding author:Tomas Skala, e-mail:tomasskala@gmail.com

\section{BACKGROUND}

There are a lot of case-reports about foreign bodies after cardiac catheterization but none was reported so far about a large newly emerged foreign-body-like structure after ablation that was just a benign fibrinous matter.

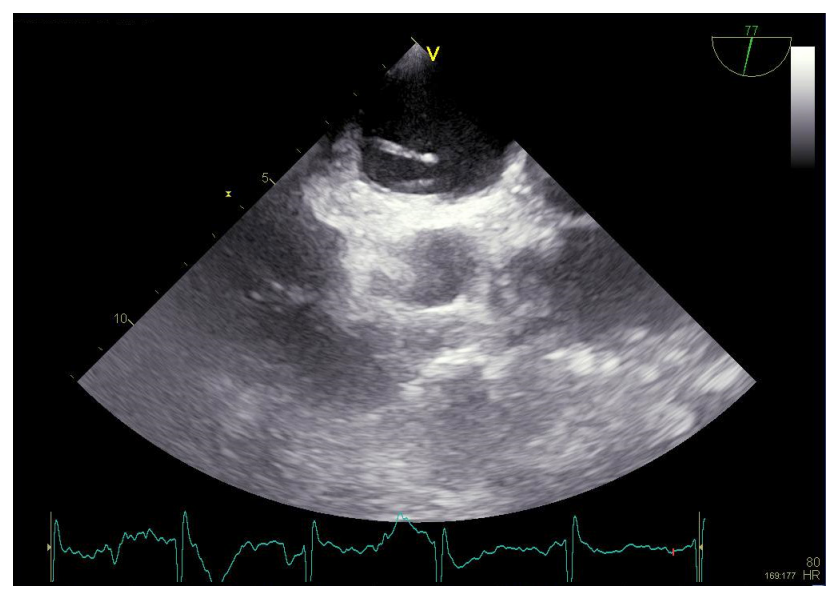

Fig. 1. Transesophageal echocardiography. A straw-like foreign body with the character of a transseptal sheath.

\section{CASE REPORT}

A 67-years old patient underwent a catheter ablation for persistent atrial fibrillation. The ablation consisted of cavotricuspid isthmus line, isolation of pulmonary veins, mitral and roof line in the left atrium (LA) and CFAE ablation. Six months after the index ablation, the patient

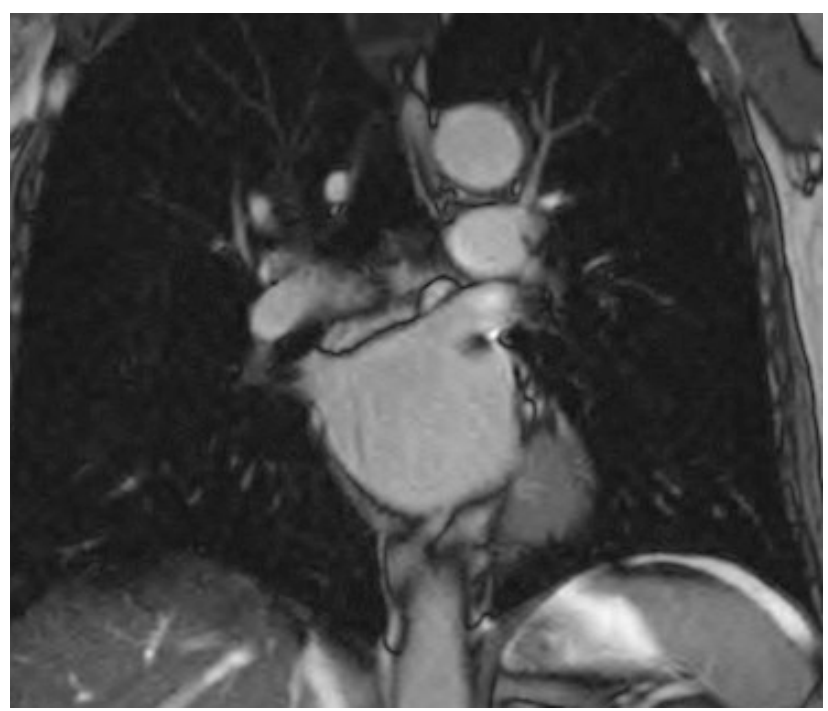

Fig. 2. Magnetic resonance imaging. A chord-like structure in the left atrium. 


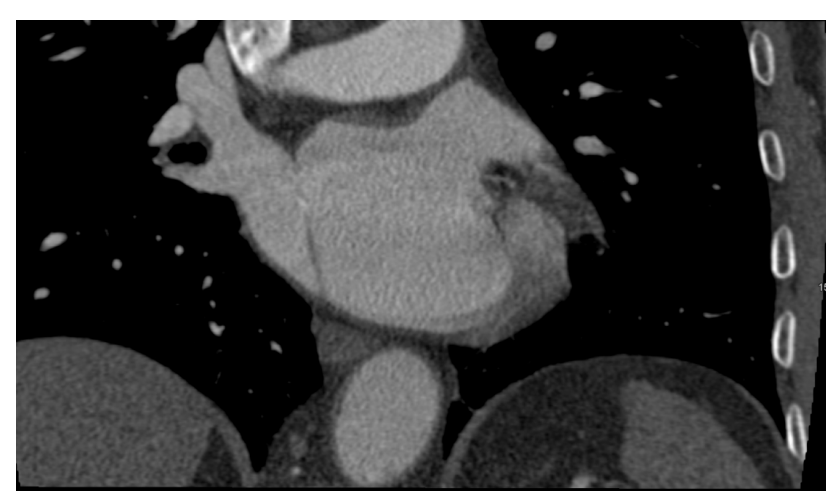

Fig. 3. Cardiac computed tomography. A chord-like structure in the left atrium.

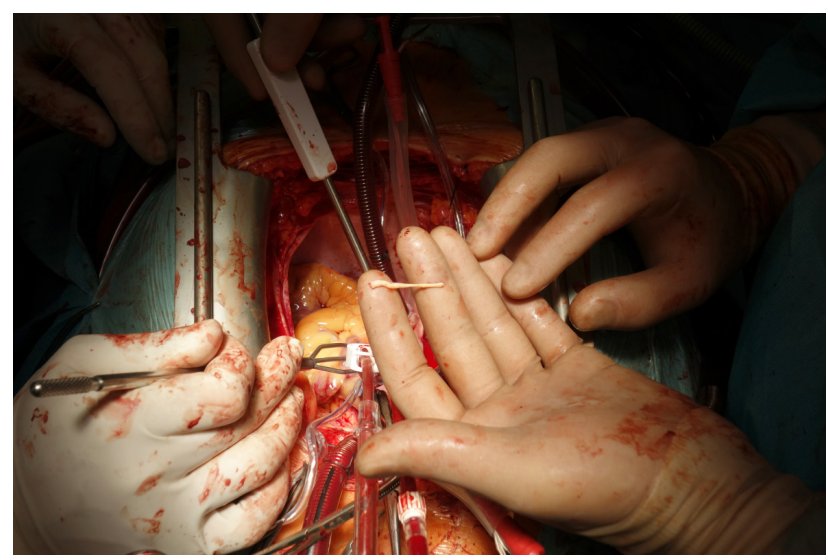

Fig. 5. Cardiac surgery. A fibrous chord-like material extirpated from the left atrium.

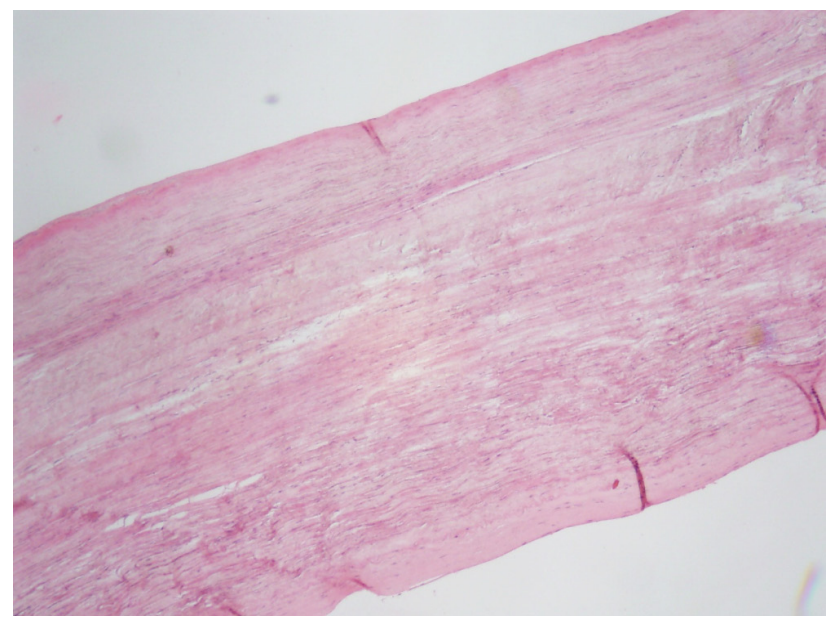

Fig. 7. Microscopy. Hematoxylin-eosin (H/E). Original magnification 200x. Myocardial tissue with continuous transition to fibrinous elastic vessel and a fibrinous tissue with focal dystrophic calcification.

presented with atrial tachycardia and was indicated for reablation.

Transesophageal echocardiography (TEE) showed a straw-like foreign body with the character of a transseptal sheath (Fig. 1). Skiagraphy showed nothing suspicious.

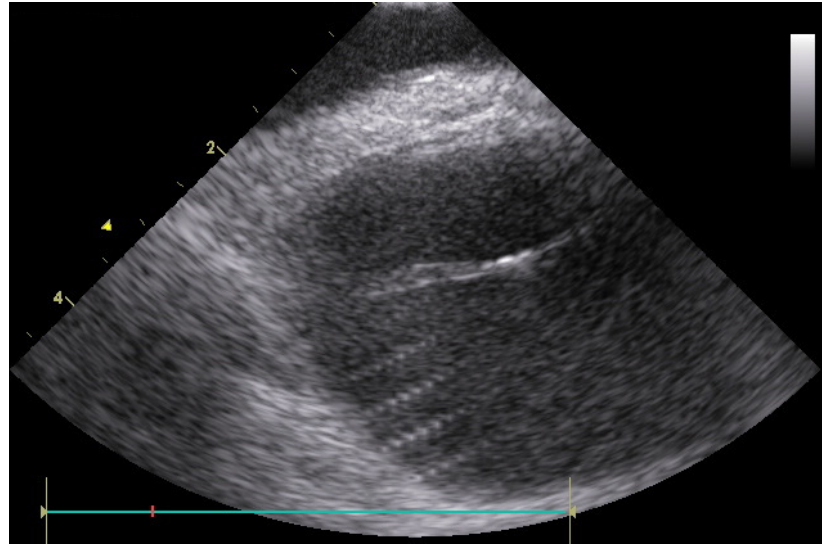

Fig. 4. Intracardiac echocardiography. A straw-like foreign body with the character of a transseptal sheath.

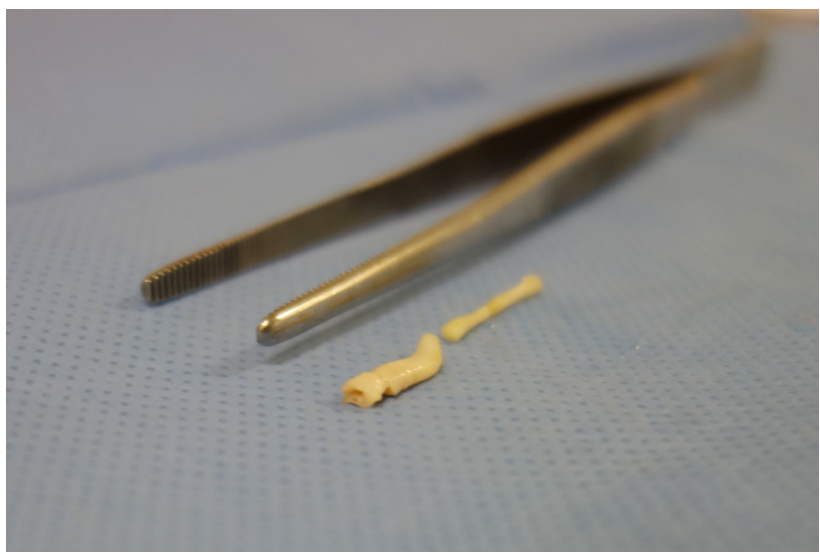

Fig. 6. Cardiac surgery. A fibrous chord-like material extirpated from the left atrium.

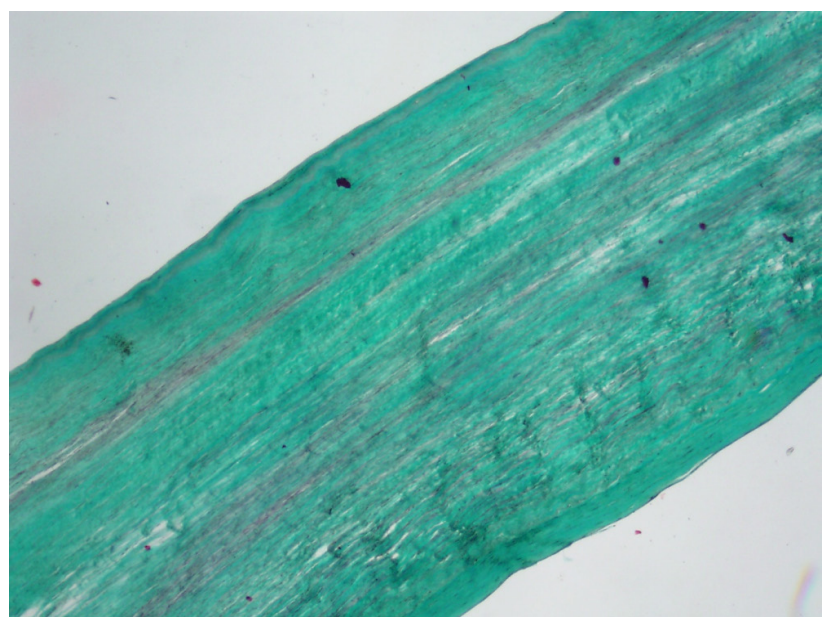

Fig. 8. Microscopy. Trichrome staining. Original magnification 200x. Green color corresponds with fibrotic tissue.

Magnetic resonance imaging (Fig. 2) and subsequent cardiac computed tomography (Fig. 3) verified a chord-like structure in LA. Cardiac catheterization was performed for final verification with the introduction of intracardiac echocardiography (ICE) to the right atrium confirming 
the TEE finding (Fig. 4). Since there was a significant suspicion of a foreign body in LA with a potential risk of cardioembolism, the patient insisted on extraction of the material. He was indicated to cardiac surgery with simultaneous Cox-Maze procedure. We found a fibrous chord-like material ( $4 \mathrm{~cm}$ long, $2.5 \mathrm{~mm}$ wide) in the LA stretched out between the right superior pulmonary vein antrum and LA wall beneath the right inferior pulmonary vein corresponding with all imaging methods (Fig. 5-6). The material was extirpated and nothing else was found in either atria. Microscopy showed myocardial tissue with continuous transition to fibrinous elastic vessel and a fibrinous tissue with focal dystrophic calcification (Fig. 7-8). No signs of foreign material were found. The patient survived with no complications and has a stable sinus rhythm after surgery.

\section{DISCUSSION}

All examinations before the first ablation, including TEE, as well as ICE during ablation, were done by the same physicians and showed no signs of foreign material. The presence of the foreign material on TEE and ICE was so striking that it could not have been omitted at the time of the index catheter ablation. This fibrinous body emerged after the first ablation. We initially thought that this emergent formation was a part of a broken transseptal sheath with a severe embolization potential. The foreign body was surprisingly invisible on fluoroscopy but its presence was clearly visible on TEE and confirmed on ICE, $\mathrm{CT}$ and MR. Thorough microscopy examination show no signs of foreign material. The origin of this fibrinous matter as well as its relation to the ablation is unclear.

\section{CONCLUSION}

A fibrinous body in left atrium mimicking foreign material emerged after a catheter ablation of atrial fibrillation. Microscopy of the extracted material showed myocardial tissue with continuous transition to fibrinous elastic vessel and a fibrinous tissue with focal dystrophic calcification.

Author contributions: All authors contributed extensively to the work presented in this paper. TS: performed catheter ablation, manuscript writing; OM: acquired ICE images; MH: acquired TEE images; ZT, MK: acquired MRI and CT images; MT: data analysing and manuscript editing.

Conflict of interest statement: None declared. 